\title{
A Home Based Guide for Hands in Rheumatoid Arthritis - An Observational Study
}

\author{
Luciana B ${ }^{1}$,Cecília C L M², Cynara F B ${ }^{2}$, Zoraida S1, Davitt $\mathbf{M}^{2}$,Manoel B B ${ }^{1}$ and \\ Eduardo de Paiva $\mathbf{M}^{2 *}$ \\ ${ }^{1}$ Department of Internal Medicine and Rheumatology, Faculty of Medical Sciences, University of Campinas-Unicamp, Campinas/SP, \\ Brazil.
}

${ }^{2}$ Prostheses and Orthoses Unit, Clinical Hospital of the State University of Campinas-HC/Unicamp, Campinas/SP, Brazil.

Received: February 19, 2018; Accepted: March 19, 2018; Published: March 26, 2018

*Corresponding author: Eduardo de Paiva Magalhães, Disciplina de Reumatologia, FCM/UNICAMP, Campinas/SP and Caixa, Postal: 6111, CEP: 13083-970, Brazil.E-mail: dreduardomagalhaes@gmail.com

\begin{abstract}
Objective: The objective of this study was to evaluate a home based program with instructions on joint protection and hand exercises on a group of patients with rheumatoid arthritis (RA).
\end{abstract}

Methods: A concise handbook with some practical instructions on joint protection and hand exercises was applied on patients with RA. Hand pain (numeric pain scale - NPS), hand function (Cochin Hand Functional Scale - CHFS), grip strength and pinch strength (dynamometer) were evaluated at the beginning of the study and after eight weeks. The subjects' comprehension, adherence and satisfaction with the intervention were evaluated at the end of protocol.

Results: Fifteen subjects were enrolled in this home-based program. After intervention there was a significant pain (NPS) reduction and hand function (CHFS) improvement $(\mathrm{p}<0.05)$.The program was considered comprehensive and easy to follow and patients referred the intention to continue following its instructions.

Conclusion: A concise home-based guide with joint protection instructions and hand exercises may help reduce pain and improve hand function. It can be a feasible and low cost adjuvant treatment in RA.

Keywords: rheumatoid arthritis, patient education, manual, exercises for hands, joint protection, occupational therapy.

\section{Introduction}

Rheumatoid arthritis (RA) is a chronic autoimmune disease characterized by persistent synovitis. Uncontrolled RA generally results in joint damage, disability and decreased quality of life. [1] Hands (proximal interphalangeal joints and metatarsophalangeal joints) and wrists are commonly affected leading to functional impairment. [2]Besides drug therapy, patient education and rehabilitation techniques are recommended since the diagnosis and throughout the treatment. [3] Occupational therapy interventions such as joint protection strategies and hand exercises are of great value, but they are not always available. $[4,5]$ Self-management educational programs may be important adjuvant instruments in the treatment of RA, especially in places with limited access to rehabilitation services. [6-8]
The objective of this study was to develop and to apply a concise home-based program for hands in RA patients.

\section{Patients and Methods}

\section{Study Design}

This study used a quasi-experimental design where patients were evaluated before and after participation in the selfmanagement program. It was conducted in the outpatient clinic of Rheumatology of the University of Campinas/Unicamp with approval of local ethics committee (CAAE 09169712.8.0000.5404) and in accordance with the Declaration of Helsinki. All patients provided a written informed consent.

\section{Subjects}

Eligible subjects were adults $>18$ years, with diagnosis of RA according to the American College of Rheumatology 1987 criteria, with stable medications over the past three months, ability to read and follow the program instructions. [9] Patients were excluded if enrolled in other rehabilitation program in the previous three months, in the presence of hand disability due other pathologies rather than RA, or if they could not perform the proposed exercises due to advanced hand deformities.

Sociodemographic (age, gender, education, income) and clinical data (disease duration, Clinical Disease Activity Index - CDAI and Health Assessment Questionnaire - HAQ) were recorded. [10-12] Subjects received the program handbook and they were instructed to read it at home, to follow the proposed recommendations and to perform the hand exercises twice a day. Motivational phone calls were carried out weekly. All of them had read and signed the informed consent form before data acquisition.

\section{The Home-Based Guide}

We developed a home based self-management program with an emphasis on hand exercises for RA patients. The program was designed so that patients could follow the program at home without assistance from a health professional. The first 
version of the program was submitted to a committee composed of two rheumatologists, three orthopedic hand surgeons and four occupational therapists regarding its clarity, coverage and relevance. Based on the committee suggestions, the program was revised.

It consisted on a handbook written in simple language with short sentences and included an introduction with brief and objective information about the disease (RA definition, main symptoms, and treatment) and joint protection (to avoid hand and wrist joints overload, to better organize and distribute their daily activities). In the second part subjects were instructed to perform six hand exercises as listed below (Figure 1). A bath sponge and one package of soft clay were given to the subjects.
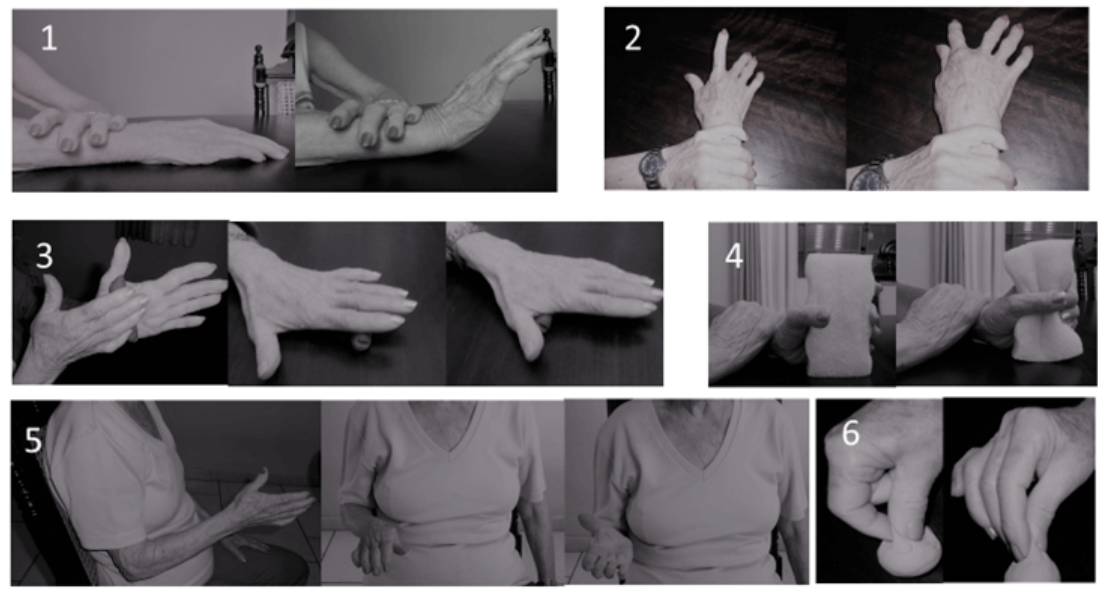

Figure 1: Hand Exercises: Active Wrist Extension (1); Collateral Finger Stretching (2); Active Forearm Pronation And Supination (3); Finger Flexion Strengthening (4); Finger Stretching (5) And Pinch Training (6).

\section{Active Wrist Extension}

Place your arm on a table, with your palm faced down and the forearm supported by the opposite hand. Raise your hand as far as you can. Hold this position for two seconds and then gently lay your hand down. Repeat this exercise 5-10 times twice a day.

\section{Collateral Finger Stretching}

Place your arm on a table, with your palm faced down and the forearm supported by the opposite hand. Now, raise your index finger, move it tour the thumb, and gently lay it down over the table. Do the same movement with the other fingers. Return to the starting position and repeat this exercise 5-10 times twice a day.

\section{Active Forearm Pronation and Supination}

With your elbow flexed and your arm resting against the side of the body, turn your palm up as far as you can. Hold this position for two seconds. Then, turn your palm down as far as you can. Hold this position for two seconds and return to the starting position. Repeat this exercise 5-10 times twice a day.

\section{Finger Flexion Strengthening}

Place your arm on a table, holding a bath sponge, with the forearm supported by the opposite hand. Tighten the sponge as much as you can. Hold this position for two seconds. Then gently open your hand releasing the sponge. Repeat this exercise 5-10 times, twice a day.

\section{Finger Stretching}

Using both hands, roll clay in the shape of a snake. Now, place the clay roll that you have made on the table and with your fingers outstretched, slide your hand over it, back and forth. Repeat this movement 10 times twice a day.

\section{Pinch Training}

squishes the clay into a ball. Remove a piece of the clay using only the thumb and the index finger. Now remove other piece of the clay ball using the thumb, the index and the middle finger. Repeat this exercise 10 times, twice a day.

\section{Outcome Measures}

Our primary outcome measures were hand pain (visual analogue scale, pain-VAS), hand function (Cochin Hand Function Scale, CHFS), grip and pinch strength.

Hand pain was accessed using a numeric pain scale ranging from 0 (no pain) to 10 (as bad as it could be). [13]

CHFS is a self-administered instrument to assess hand function. It contains 18 items regarding hand ability in the kitchen, during dressing, while performing personal hygiene, while performing office tasks, and other general items. Each question is scored from 0 (no difficulty) to 5 (impossible to do). A total CHFS score is obtained by adding the scores from all questions (range 0-90). [14]The Brazilian version was used. [15]

The grip strength and pinch strength was measured with a hydraulic hand-grip dynamometer (Jamar®) and a pinchdynamometers (Pinch Gauges $®$ ) respectively, using standard protocols. [16] The peak force produced by the dominant hand (Kgf) was used for analysis. 
At the end of the protocol subjects impressions about the program were checked by asking their opinion about the following statements: "The manual's instructions were easy to understand", "According to the manual's instructions, I modified the way I perform daily activities", "I am pleased with the results obtained using the manual", "I intend to continue doing these exercises after the end of the study". In each question patients were asked to mark with an " $\mathrm{x}$ " in the item that better represented his/her opinion: 1: strongly disagree, 2: partially disagree, 3 : indifferent, 4: partially agree, and 5 : totally agree.

An exercise diary was included in the end of the handbook. Subjects were asked to check in a calendar each day he/she performed the exercises.

Patients were also asked to inform about the occurrence of any adverse effects related to hand exercises.

\section{Statistical Analysis}

Data were entered into an Excel database (Windows) and then transferred to The SAS System for Windows, version 9.4 (SAS Institute Inc., Cary, NC, USA) for analysis. Descriptive statistics consisting of mean, median, maximum and minimum values, and standard deviations (SD) were computed for all demographic characteristics and outcome measures. Pre and post intervention differences were calculated for the outcome measures (NPS, CHFS, the grip strength and pinch strength). For comparisons between different times ( 0 and 8 weeks), analysis of variance (ANOVA) for repeated measures were performed. Because of the absence of a normal distribution, variables were transformed into ranks.

Statistical significance was set at $\mathrm{p} \leq 0.05$.

\section{Results}

Fifteen patients were evaluated (14 women and 1 man) with mean age of 56 years $(42-67, \pm 8.2)$, mean duration of disease of 14 years $(3-44, \pm 10.23)$, average education level of 7.5 years $(4-15, \pm 4$ ) average monthly income of R\$1,254.84 ( $\sim$ US\$ 400) mean HAQ of $0,9(0-1.88, \pm 0.62)$ and CDAI of $24.41(5-49, \pm$ 11.05).Nine patients were taking methotrexate alone, three were taking methotrexate and hydroxychloroquine, one was taking leflunomide and two were taking methotrexate and adalimumab. Medication doses were stable in the last three months.

Table 1 shows the values of pain (NPS), hand function (CHFS), grip and pinch strength. There was a significant reduction of pain and improvement of CHFS. No significant change was noted for grip and pinch strength. (Table 1)

Table 1: Values of pain in the hands (pain-VAS), Cochin Hand Function Scale, pinch strength and grip strength $(\mathrm{kg} / \mathrm{f})$ in initial evaluation and after 8 weeks using the manual.

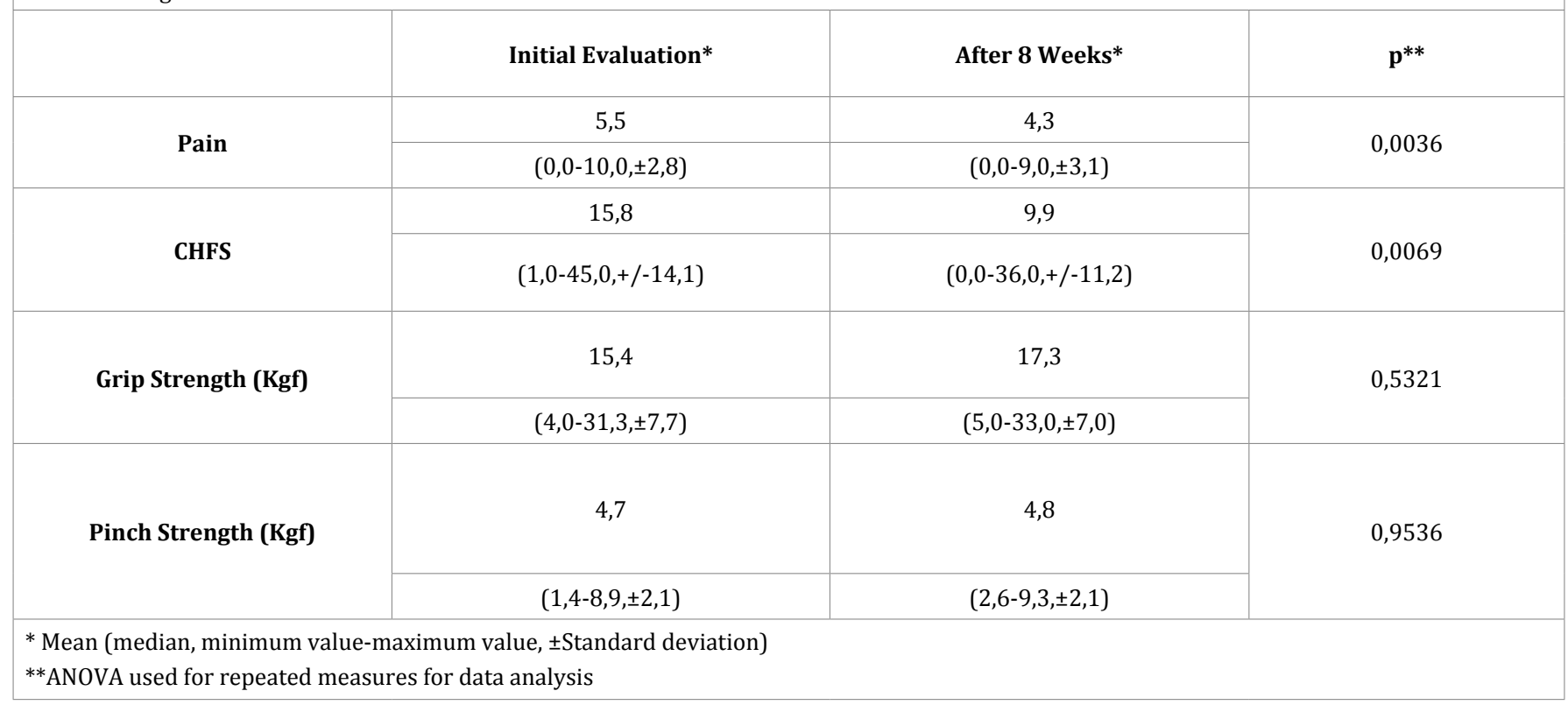

All patients totally agreed that program instructions were easy to understand, that after its use they modified the way they performed their daily activities, that they were pleased with the results of this intervention and that they intended to continue doing the exercises after the end of the study.

The practice of the exercises was about 6 times a week.

Patients did not complain about pain or any other adverse events related to hand exercises.

\section{Discussion}

Patient education and rehabilitation have been recommended in the treatment of RA since its diagnosis and throughout the treatment.[3] Unfortunately, rehabilitation is not always available due several reasons such as the distance from patients to the rehabilitation center, the high no-show rate due personal reasons or disease related problems, difficulties in attending chronic patients regularly, etc. In this context, home-based educational 
programs are known to be a high-available and low-cost rehab strategy. [17]

In this study, a home-based program was developed and applied on a group of rheumatoid patients. Joint protection was addressed in the first part of the handbook. Patients were asked to avoid hand and wrist joints overload, to better organize and distribute their daily activities. Hand exercises were the main program strategy. They were proposed to be easy to practice, without the need of face-to-face sessions or a health professional supervision. After eight weeks, we observed pain reduction and hand function improvement. These findings may have resulted from the increased attention given to hand care as well as to the hands exercises. There was no significant difference regarding grip and pinch strength. Pain and function may improve earlier than strength. The short follow-up time may, at least in part, justify the non-significant grip and pinch strengthening.

Previous studies have shown the benefits of hand exercises in rheumatoid patients. Brighton et al. observed significant grip strength improvement in a group of 44 female patients after a 48-month controlled trial with hand exercises. [18] Also, Brorsson et al. reported hand force and function improvement studying 40 RA patients in a 6-weeks controlled trial. [19] Cima et al., studying the effect of hand exercises in a controlled trial with 20 women with hand deformities, observed hand strengthening and function improvement after ten weeks. [20] In 2015, a multicenter controlled trial with 438 RA subjects obtained hand strengthening and function improvement with a home-based exercise program. They have also observed a reduction in the number of swollen and tender joints. [21, 22] All of these programs were supported by face-to-face sessions, with the assistance of a health professional. In Brazil, occupational therapy care for rheumatic diseases is unusual due to the low availability of these professionals in the public health system. Therefore, our objective was to apply the manual alone without face-to-face sessions so that it could be widely used, both in outpatient clinic as well as in primary care, without the need of a trained professional. This is the most important advantage of this intervention. Although it is uncertain if its benefits would be sustained when applied in a larger group of subjects during a longer period, it was well accepted and practiced by patients not used to this kind of intervention.

This is study has several limitations. It was applied in a small number of subjects during a short period of follow-up. Since it was not a controlled trial, we cannot rule out a placebo effect. Sustained adherence and benefits over longer periods of time are also still to be determined. A self-reported instrument was used to assess the hand function and it would be interesting to use another objective tool. We did not assess how subjects were performing the exercises, so we cannot assure that they were done correctly.

\section{Conclusion}

A concise home-based program with joint protection instructions and hand exercise resulted in pain reduction and hand function improvement during a short period of follow-up.
Further controlled studies with a higher number of subjects are necessary to confirm its efficacy over longer periods of time.

\section{Acknowledgments}

Heitor J. R. Ulson, Marcos Felipe Marcato de Abreu, Juliana F. Sekiyama, Mauro Bosi, Marco Antonio de Carvalho,Pola Maria Poli de Araújo, Leila Maria Abdalla, Cynara Ferreira Bernardes e Fernanda Bittencourt Mourafor taking part on the manual judgment committee.

\section{Disclosure Statement}

The authors have declared no conflicts of interest

\section{References}

1. Scott DL, Wolfe F, Huizinga TW. Rheumatoid arthritis. Lancet. 2010; 376(9746):1094-1108. doi: 10.1016/S0140-6736(10)60826-4

2. Chung KC, Pushman AG. Current concepts in the management of the rheumatoid hand. J Hand Surg Am. 2011; 36(4):736-747. doi: 10.1016/j.jhsa.2011.01.019

3. da Mota LM, Cruz BA, Brenol CV, Pereira IA, Rezende-Fronza LS, Bertolo MB, et al. 2012 Brazilian Society of Rheumatology Consensus for the treatment of rheumatoid arthritis. Rev Bras Reumatol. 2012; 52(2):152-174.

4. Hammond A, Young A, Kidao R. A randomised controlled trial of occupational therapy for people with early rheumatoid arthritis. Ann Rheum Dis. 2004; 63(1):23-30. doi: 10.1136/ard.2002.001511

5. Hammond, A. What is the role of the occupational therapist? Best Pract Res Clin Rheumatol. 2004; 18(4): 491-505. doi:10.1016/j. berh.2004.04.001

6. Hammond A, Lincoln N, Sutcliffe L. A crossover trial evaluating an educational-behavioural joint protection programme for people with rheumatoid arthritis. Patient Educ Couns. 1999; 37(1):19-32.

7. Hammond A, Nierdermann K. Patient education and self management. In: Dziedzic K, Hammond A. Rheumatology Evidence-based practice for physiotherapists and occupational therapistis. Elsevier; 2010:7797.

8. Manning VL, Hurley MV, Scott DI, Coker B, Choy E, Bearne LM. Education, self-management, and upper extremiity exercise training in people with rheumatoid arthritis: a rondomized controlled trial. Arthritis Care Res (Hoboken). 2014; 66(2):217-227. doi: 10.1002/ acr.22102

9. Arnett FC, Edworthy SM, Bloch DA, McShane DJ, Fries JF, Cooper NS et al. The American Rheumatism Association 1987 revised criteria for the classification of rheumatoid arthritis. Arthritis Rheum 1988; 31:315-324.

10. Smolen JS, Aletaha D. Scores for all seasons: SDAI and CDAI. Clin Exp Rheumatol. 2014; 32(5 Suppl 85): S-75-9.

11. Fries JF, Spitz P, Kraines RG, Holman HR. Measurement of patient outcome in arthritis. Arthritis Rheum. 1980; 23(2):137-145.

12. Ferraz MB, Oliveira LM, Araújo PM, Atra E, Tugwell P. Crosscultural reliability of the physical ability dimension of the heath assessment questionnaire. J Rheumatol. 1990; 17(6):813-817. 
13. Hawker GA, Mian S, Kendzerska T, French M. Measures of adult pain: Visual Analog Scale for Pain (VAS Pain), Numeric Rating Scale for Pain (NRS Pain), McGill Pain Questionnaire (MPQ), Short-Form McGill Pain Questionnaire (SF-MPQ), Chronic Pain Grade Scale (CPGS), Short Form-36 Bodily Pain Scale (SF-36 BPS), and Measure of Intermittent and Constant Osteoarthritis Pain (ICOAP). Arthritis Care Res (Hoboken). 2011; 63 Suppl 11:S240-252. doi: 10.1002/acr.20543

14. Duruoz MT, Poiraudeau S, Fermanian J, Menkes CJ, Amor B, Dougados $\mathrm{M}$, et al. Development and validation of a rheumatoid hand functional disability scale that assesses functional handicap. J Rheumatol 1996; 23(7):1167-1172.

15. Chiari A, Sardim CC, Natour J. Translation, cultural adaptation and reproducibility of the Cochin Hand Functional Scale Questionnaire for Brazil. Clinics (Sao Paulo). 2011; 66:731-736.

16. Fess EE, Moran C. Clinical assessment recommendations. Indianapolis: American Society of Hand therapists Monograph; 1981.

17. Manning VL, Kaambwa B, Ratcliffe J, Scott DL, Choy E, Hurley MV, et al. Economic evaluation of a brief education, self-management and upper limb exercise training in people with rheumatoid arthritis (EXTRA) programme: a trial-based analysis. Rheumatology (Oxford). 2015; 54(2):302-309. doi: 10.1093/rheumatology/keu319
18. Brighton SW, Lubbe JE, van der Merwe CA. The effect of a long-term exercise programme on the rheumatoid hand. $\mathrm{Br} J$ Rheumatol. 1993; 32(5):392-395.

19. Brorsson S, Hilliges M, Sollerman C, Nilsdotter A. A six-week hand exercise programme improves strength and hand function in patients with rheumatoid arthritis. J Rehabil Med. 2009; 41(5):338-342. doi: 10.2340/16501977-0334

20. Cima SR, Barone A, Porto JM, Abreu DCC. Strengthening exercises to improve hand strength and functionality in rheumatoid arthritis with hand deformities: a randomized, controlled trial. Rheumatol Int. 2013; 33(3):725-732. doi: 10.1007/s00296-012-2447-8

21. Williams MA, Williamson EM, Heine PJ, Nichols V, Glover MJ, Dritsaki $\mathrm{M}$, et al. Strengthening and stretching for Rheumatoid Arthritis of the Hand (SARAH). A randomised controlled trial and economic evaluation. Health Technol Assess. 2015; 19(19):1-222. doi: 10.3310/ hta19190

22. Lamb SE, Williamson EM, Heine PJ, Adams J, Dosanjh S, Dritsaki M, et al. Exercises to improve function of the rheumatoid hand (SARAH): a randomised controlled trial. Lancet. 2015; 385(9966):421-429.doi: 10.1016/S0140-6736(14)60998-3 